\title{
Radiologic Impression
}

National Cancer Institute

\section{Source}

National Cancer Institute. Radiologic Impression. NCI Thesaurus. Code C122081.

The summary or conclusion of the most important radiologic findings. 